Original paper

\title{
Experimental optimisation of the X-ray energy in microbeam radiation therapy
}

\author{
Jayde Livingstone $^{\mathrm{a}, *}$, Andrew W. Stevenson ${ }^{\mathrm{a}}$, Daniel Häusermann ${ }^{\mathrm{a}}$, Jean-François Adam ${ }^{\mathrm{b}, \mathrm{c}}$ \\ a Australian Synchrotron, Australian Nuclear Science and Technology Organisation, 800 Blackburn Road, Clayton, Victoria, Australia \\ ${ }^{\mathrm{b}}$ Equipe d'accueil Rayonnement Synchrotron et Recherche Médicale, Université Grenoble-Alpes, Grenoble, France \\ ${ }^{\mathrm{c}}$ Centre Hospitalier Universitaire de Grenoble, Grenoble, France
}

\section{A R T I C L E I N F O}

\section{Keywords:}

Diamond detectors

Microbeam radiation therapy

Synchrotron

\begin{abstract}
A B S T R A C T
Microbeam radiation therapy has demonstrated superior normal tissue sparing properties compared to broadbeam radiation fields. The ratio of the microbeam peak dose to the valley dose (PVDR), which is dependent on the X-ray energy/spectrum and geometry, should be maximised for an optimal therapeutic ratio. Simulation studies in the literature report the optimal energy for MRT based on the PVDR. However, most of these studies have considered different microbeam geometries to that at the Imaging and Medical Beamline $(50 \mu \mathrm{m}$ beam width with a spacing of $400 \mu \mathrm{m}$ ). We present the first fully experimental investigation of the energy dependence of PVDR and microbeam penumbra. Using monochromatic X-ray energies in the range 40-120 keV the PVDR was shown to increase with increasing energy up to $100 \mathrm{keV}$ before plateauing. PVDRs measured for pink beams were consistently higher than those for monochromatic energies similar or equivalent to the average energy of the spectrum. The highest PVDR was found for a pink beam average energy of $124 \mathrm{keV}$. Conversely, the microbeam penumbra decreased with increasing energy before plateauing for energies above $90 \mathrm{keV}$. The effect of bone on the PVDR was investigated at energies 60, 95 and $120 \mathrm{keV}$. At depths greater than $20 \mathrm{~mm}$ beyond the bone/water interface there was almost no effect on the PVDR. In conclusion, the optimal energy range for MRT at IMBL is $90-120 \mathrm{keV}$, however when considering the IMBL flux at different energies, a spectrum with $95 \mathrm{keV}$ weighted average energy was found to be the best compromise.
\end{abstract}

\section{Introduction}

Spatial fractionation in the context of radiotherapy is the practice of collimating a beam into an array of smaller beams to deliver a non-homogeneous distribution of radiation dose to the target. It is a concept that was first introduced to radiotherapy more than half a century ago via grid therapy [1]. Grid therapy was at first performed using orthovoltage X-ray tubes but later transferred to megavoltage (MV) linacs $[2,3]$. In grid therapy, the beam is collimated into smaller beams $(1 \mathrm{~cm}$ wide) to allow delivery of high doses to deep-seated tumours whilst minimising damage to skin [4], thus enhancing the therapeutic ratio. The tissue sparing effects of microbeams were first shown in the $1960 \mathrm{~s}$ using $25 \mu \mathrm{m}$ wide deuteron beams to simulate heavy cosmic ray particles $[5,6]$. When compared with $1 \mathrm{~mm}$ wide deuteron beams, it was found that tissues and cells irradiated with the microbeam could withstand much higher doses before cell death was observed. This illustrates that the tissue sparing effect increases with decreasing irradiation volume. Since the 1990 s synchrotron microbeam radiation therapy has been under development and continued to demonstrate a sparing effect in many normal tissues [7-15]. Radiation resistant brain tumours, particularly in infants, have been identified as a suitable target patient group for MRT due to the ability to deliver extremely high doses without causing significant damage to the surrounding healthy brain [16-18].

Various irradiation geometries, based on different ratios of irradiated and "unirradiated" tissue, have been theoretically and experimentally investigated in an attempt to optimise the therapeutic ratio [19-22]. Based on the findings of these investigations, the Imaging and Medical Beamline (IMBL) at the Australian Synchrotron has settled on a fixed geometry of $50 \mu \mathrm{m}$ wide microbeams separated (centre-to-centre) by $400 \mu \mathrm{m}$.

Kilovoltage synchrotron wiggler generated $\mathrm{x}$-rays are ideal for creating such arrays of microbeams as the small lateral range of secondary electrons results in a very steep dose gradient between the peaks and the regions of lower dose, "valleys" between the peaks. The low beam divergence also allows the microbeams to be preserved as the beam penetrates absorbing material. Since the valley dose is believed to be linked to the normal tissue tolerance $[10,23]$, it is important to

\footnotetext{
* Corresponding author.

E-mail address: Jayde.Livingstone@synchrotron.org.au (J. Livingstone).
} 
maintain a low valley dose and high peak-to-valley-dose ration, or PVDR. The most important disadvantage of $\mathrm{kV}$ photons is the reduced penetration in matter. For deep-seated tumours, it may thus be necessary to make a compromise between PVDR and beam penetration. A number of Monte Carlo based investigations have been performed to characterise the energy dependence of the PVDR [20,24-28]. Shinohara et al. [28] made a recommendation on the optimal energy range, 100-300 keV, for MRT treatment of deep-seated tumours and Prezado et al. [27] found the optimal energy to be $175 \mathrm{keV}$. However, of the studies mentioned, only one [27] is based on the same microbeam geometry used at IMBL. Furthermore, to the knowledge of the authors, no experimental investigation of the energy dependence of the PVDR has been performed. Experimental data is required to verify the simulation results.

This study aims to present the optimal energy range for synchrotron MRT based on measurements of PVDR and microbeam penumbra as a function of beam energy. Given the interest in brain tumours as a clinical case for MRT, the effect of bone on the PVDR at depth has been investigated using a simple phantom to approximate a human head.

\section{Materials and methods}

\subsection{X-ray source}

This study was performed using the preclinical radiotherapy instrumentation in Hutch 2B of the Imaging and Medical Beamline (IMBL) at the Australian Synchrotron [29].

Both polychromatic and monochromatic X-ray beams were used in this investigation of microbeam peak to valley dose ratio as a function of X-ray energy. The IMBL X-ray source is a superconducting multipole wiggler with a maximum operating magnetic field of $4.2 \mathrm{~T}$ and typical operating field of 3.0 T. The latter was used for this study. The wiggler produces a high flux of $\mathrm{x}$-rays in the $\mathrm{kV}$ energy range, peaking around $20 \mathrm{keV}$. Filtering the X-ray beam using metallic foils such as copper, aluminium and molybdenum changes the shape of the spectrum. Different combinations of filters can be inserted in the beam to give the desired mean energy or X-ray flux. Filtered beams are referred to as "pink" beams. The program spec.exe described by Stevenson et al.[30] was used to calculate parameters such as the peak energy, weighted mean energy and half-value layer for given combinations of filters or pink beams. A summary of the filtrations used and the corresponding parameters is given in Table 1 and a graphical representation of the spectra is given in Fig. 1. Monochromatic beams in the range $40-120 \mathrm{keV}$ were selected from the $\mathrm{Al}-\mathrm{Al}$ filtered spectrum using the silicon crystal monochromator. The energy resolution $(\Delta E / E)$ of the IMBL monochromator is of the order of $10^{-3}$. Pink beams with weighted average energies of $82.9,95.1$ and $124 \mathrm{keV}$ were used in the study.

Microbeams are produced by a multislit collimator (Usinage et Nouvelles Technologies, Morbier, France), which collimates the beam into 125 vertical microbeams of $50 \mathrm{~mm}$ width with a $400 \mathrm{~mm}$ centre-tocentre (c-t-c) spacing [29]. Tungsten carbide layers of $350 \mathrm{~mm}$ are sandwiched together to create a $50 \mathrm{~mm}$ spacing between them. The tungsten carbide layers are $8 \mathrm{~mm}$ thick in the beam direction. The multislit collimator is housed in an aluminium box (fabricated at the Australian Synchrotron) which is flushed with helium gas and water-

Table 1

Peak energy, weighted average energy and aluminium half-value layers for the spectra produced by each filtration combination.

\begin{tabular}{llll}
\hline Filter name & Peak energy (keV) & Weighted ave. energy (keV) & HVL Al (mm) \\
\hline Al-Al & 47.8 & 55.0 & 6.27 \\
$\mathrm{Al}-\mathrm{Cu}$ & 76.2 & 82.9 & 11.9 \\
$\mathrm{Cu}-\mathrm{Cu}$ & 87.5 & 95.1 & 13.6 \\
AlMo-AlMo & 117 & 124 & 16.5 \\
\hline
\end{tabular}

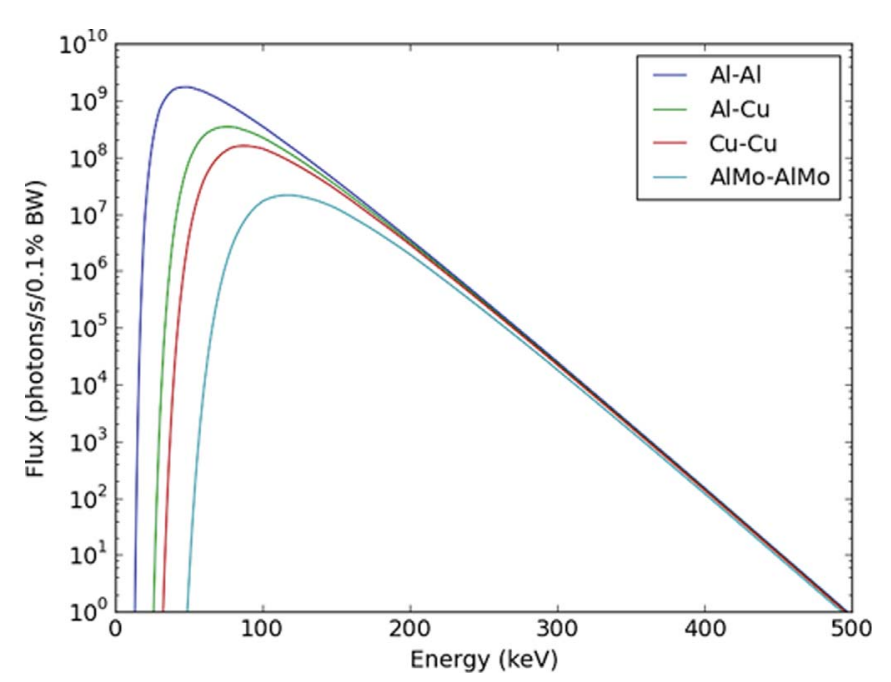

Fig. 1. The spectra produced by the different filtration combinations given in Table 1.

cooled. Kapton entrance and exit windows minimise beam perturbance. For alignment with the beam, the collimator was rotated about the $z$ (vertical) axis. The current recorded from a free-air ionisation chamber immediately downstream of the collimator was simultaneously recorded, and the angle of the collimator (with respect to the vertical axis) was set to the angle at which the maximum current was recorded.

\subsection{Measurements}

Dosimetric measurements were performed using a PTW (Freiburg, Germany) microDiamond 60019 detector read-out using a PTW Unidos Webline electrometer. Lateral profiles were acquired over the central $1.2 \mathrm{~mm}$ (corresponding to three microbeam peaks and two valleys) of the beam at various depths in a water phantom using the method described in [31]. The PVDR was calculated using the mean of the three peak doses and the mean of the doses in the central $100 \mu \mathrm{m}$ of the two valleys. For a graphical representation of the points used to calculate the PVDR in an example profile exhibiting four peaks and three valleys, see $\S 2.3$ of [29]. The PVDR was measured for monochromatic energies between 40 and $120 \mathrm{keV}$ at depths of 5, 10, 20 and $50 \mathrm{~mm}$ in a water tank. As MRT studies are typically performed using a pink beam, the relationship between PVDR and pink beam energy (82.9, 95.1 and $124 \mathrm{keV}$ ) was also measured at the same measurement depths. Percentage depth dose curves (PDD) were also acquired in the water tank for beams of different energies.

In addition to comparing PVDRs at different energies, the penumbra, defined as the lateral distance between the $80 \%$ and $20 \%$ of maximum dose points on one side of a beam profile [32], was also measured as a function of energy. The penumbra was measured on each side of the central microbeam from the lateral profile acquired at each monochromatic energy. This measurement was repeated on profiles acquired at different depths in water (between $5 \mathrm{~mm}$ and $50 \mathrm{~mm}$ ). As it was observed that the penumbra does not change with depth in this range, a mean and standard deviation was calculated using the measurements at different depths.

Radioresistant brain tumours have been identified as a clinical case for MRT. As a result of this, most of the preclinical MRT survival data exists for small animal brain tumour models. To simulate a clinically relevant scenario, Gammex (Middleton, USA) inner bone (456) equivalent material was positioned at the entrance of the water tank to approximate an adult male head. The thickness of bone material used was $8 \mathrm{~mm}$, which closely approximates the average thickness of the frontal and occipital regions of the adult male skull [33]. Lateral profiles and PVDRs were obtained using the same method described above and PVDRs were compared to those obtained in the absence of bone 


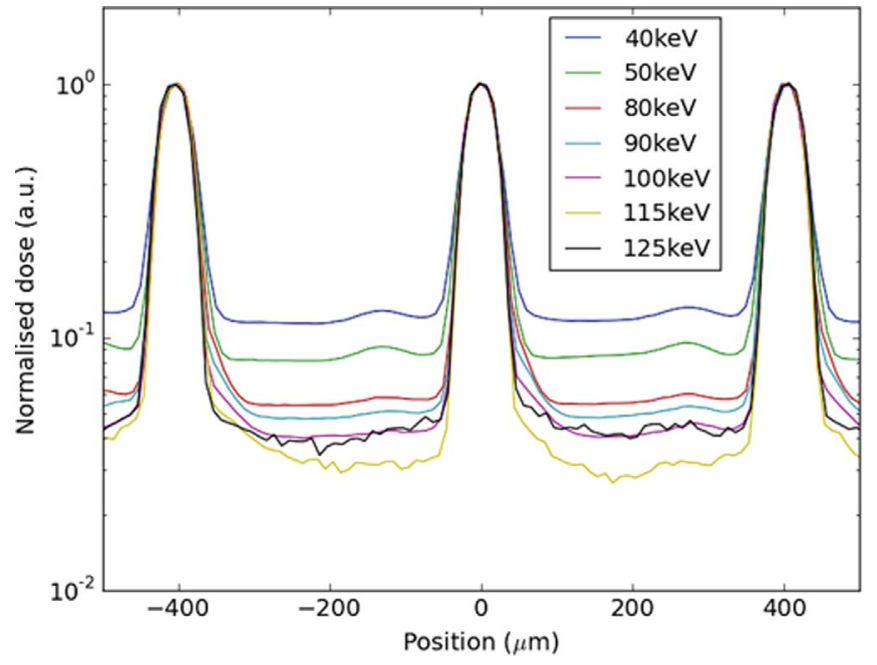

Fig. 2. Profiles of the central microbeam peaks at various monochromatic X-ray energies. Each profile has been normalised to the peak dose (averaged over three peaks). The profiles were acquired at $20 \mathrm{~mm}$ depth in a water tank.

material.

\section{Results}

Lateral profiles obtained using monochromatic beams are displayed in Fig. 2. For clarity, only a subset of all the monochromatic energies studied are displayed. The profiles have been normalised to the peak dose at each energy, where the peak dose is calculated from the mean dose across the three microbeams. The beam energy clearly has a strong influence on the ratio of peak to valley dose, or PVDR. One can qualitatively observe that the PVDR is increasing with increasing beam energy up to $115 \mathrm{keV}$, above which it appears to decrease again. Percentage depth dose curves for a subset of monochromatic energies and the $95 \mathrm{keV}$ average energy spectrum are shown in Fig. 3. The curves, which are normalised at $5 \mathrm{~mm}$ depth clearly illustrate that higher energy beams are more penetrating in matter. Also, the PDD for $95 \mathrm{keV}$ average energy matches the PDD for $95 \mathrm{keV}$ monochromatic beam within the uncertainty.

Values of PVDR as a function of monochromatic beam energy are plotted in Fig. 4 for measurement depths of 5, 10, 20 and $50 \mathrm{~mm}$ in a water tank. It is again clear that the PVDR is increasing with increasing beam energy up to at least $100 \mathrm{keV}$. Beyond this energy, due to the

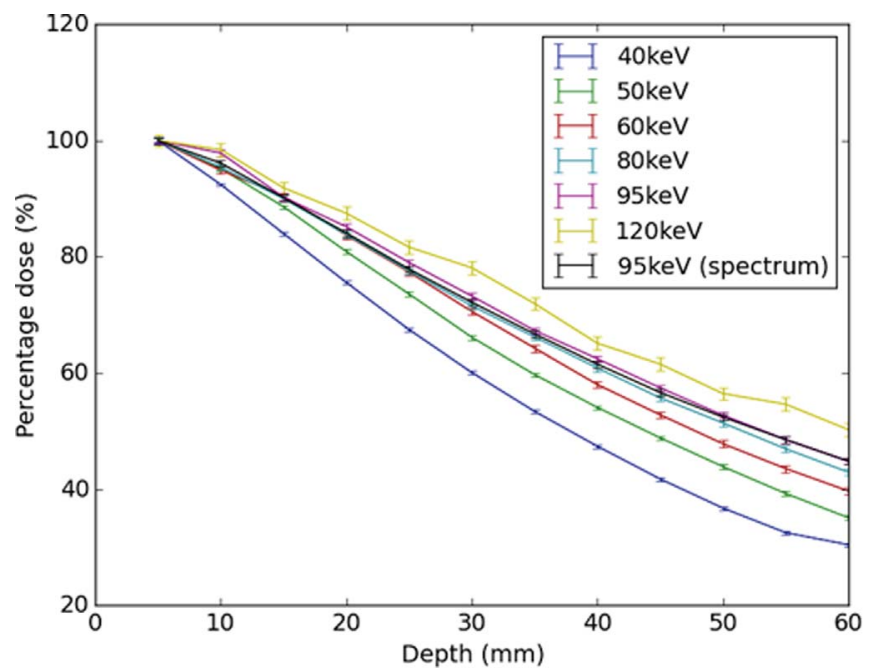

Fig. 3. Percentage depth dose curves, normalised at $5 \mathrm{~mm}$ depth in water, for different monochromatic X-ray energies.

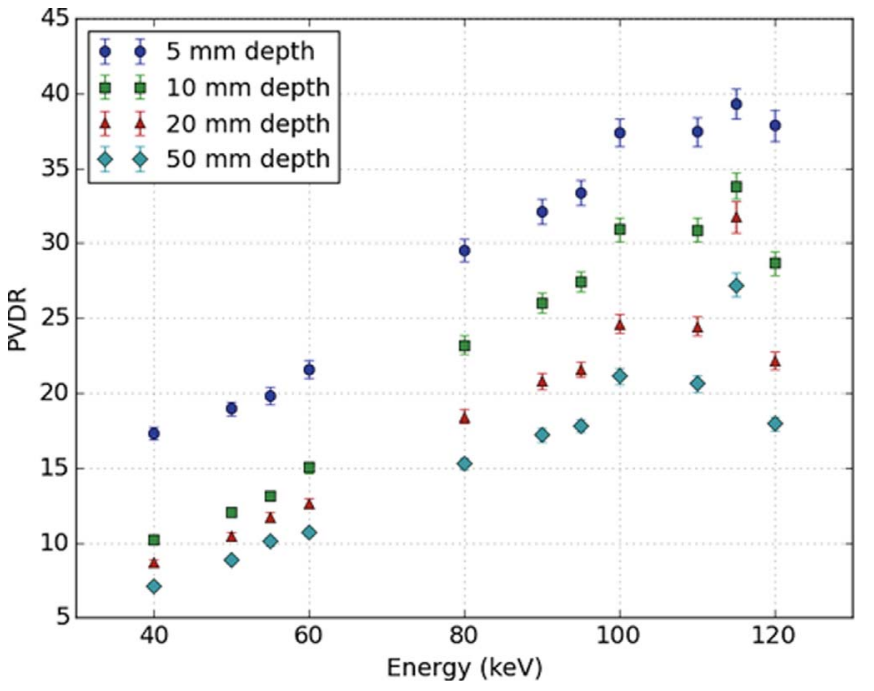

Fig. 4. PVDR as a function of monochromatic X-ray energy.

scattered data, larger uncertainties and lack of information for energies above $120 \mathrm{keV}$, it is difficult to comment on the trend. At best, the PVDR continues to increase up to $115 \mathrm{keV}$, or at worst, it could be considered to plateau after $100 \mathrm{keV}$. The lack of data above $120 \mathrm{keV}$ is due to physical limitations in monchromator crystal rotations and separation. The larger uncertainties at the higher energies is due to the rapid decrease in flux causing statistical fluctuations in the measurements.

The relationship between PVDR and pink beam average energy is shown in Fig. 5 for the same measurement depths. In Fig. 6, PVDR as a function of weighted average energy are compared to the PVDR as a function of the closest monochromatic energy. Close to the surface of the water tank ( $\leqslant 10 \mathrm{~mm}$ ), the PVDR for a given monochromatic energy is similar, within experimental uncertainty, to that of the equivalent average energy. With increasing depth, the difference between the PVDRs increases, with the PVDR for pink beams being consistently higher than those for equivalent monochromatic beams due to beam hardening.

The microbeam penumbra as a function of monochromatic beam energy are shown in Fig. 7. The penumbra were measured using the lateral profiles acquired at each energy and at each measurement depth. The penumbra was found to be constant with depth between 5 and $50 \mathrm{~mm}$ and the values presented represent a mean of the four values

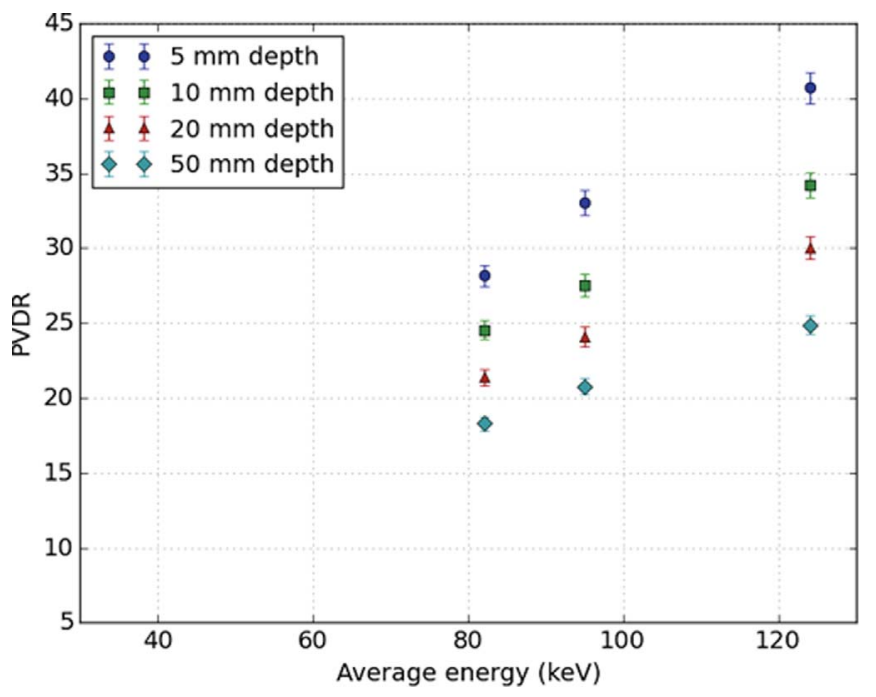

Fig. 5. PVDR as a function of weighted average energy of X-ray spectra. 


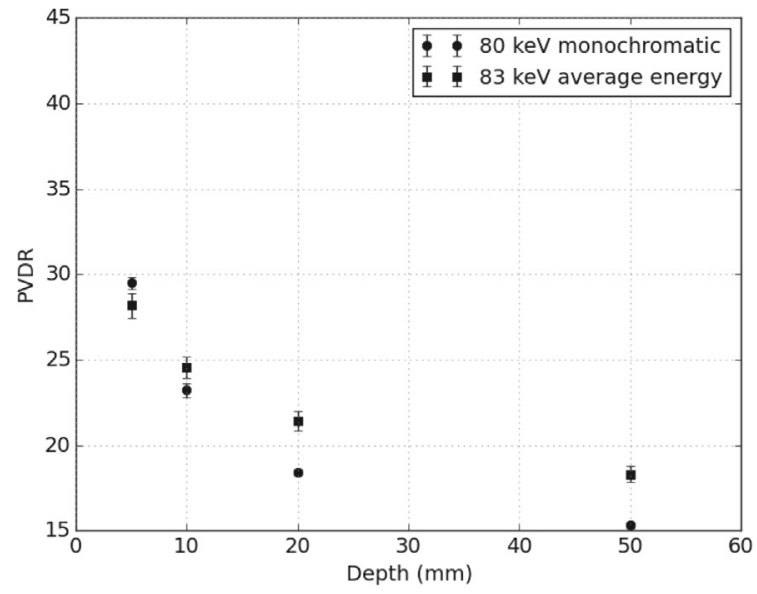

(a)

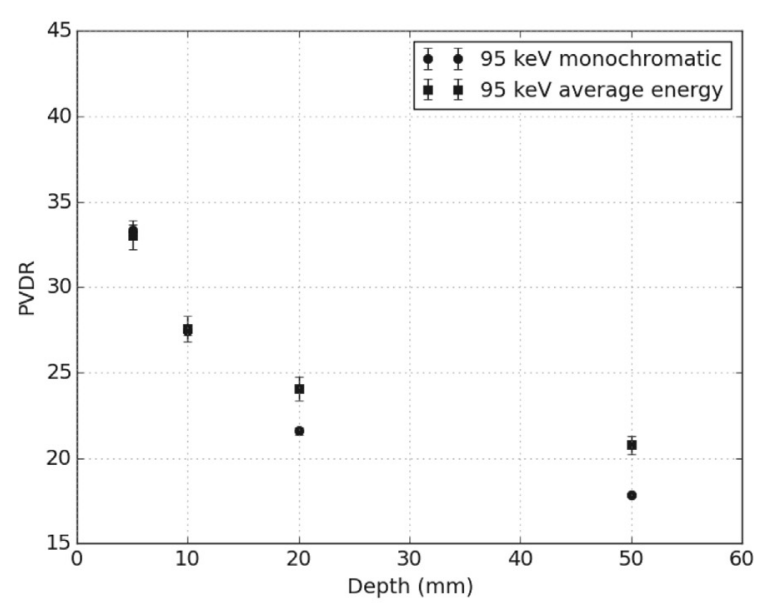

(b)

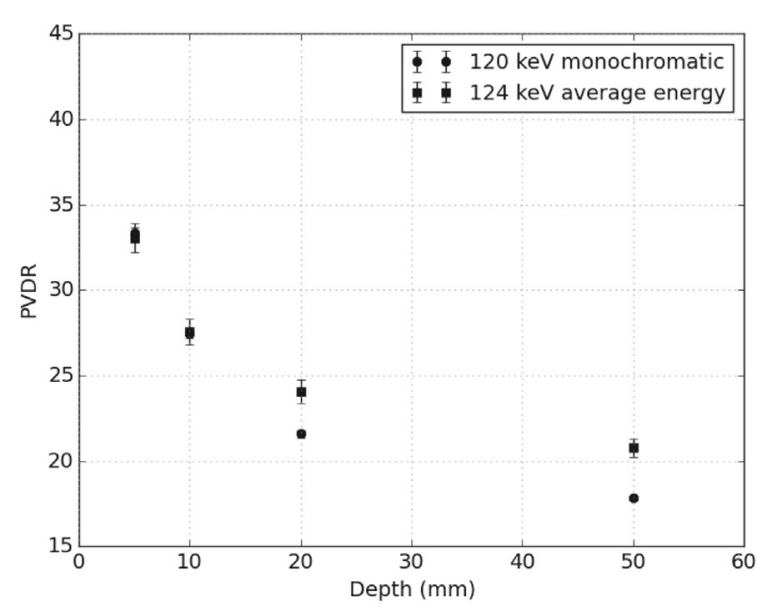

(c)

Fig. 6. Comparison of PVDR vs. depth curves measured in monochromatic beams and spectra with approximately equivalent average energy: (a) $80 \mathrm{keV}$ monochromatic compared to $83 \mathrm{keV}$ average energy, (b) $95 \mathrm{keV}$ monochromatic compared to $95 \mathrm{keV}$ average energy and (c) $120 \mathrm{keV}$ monochromatic compared to $124 \mathrm{keV}$ average energy.

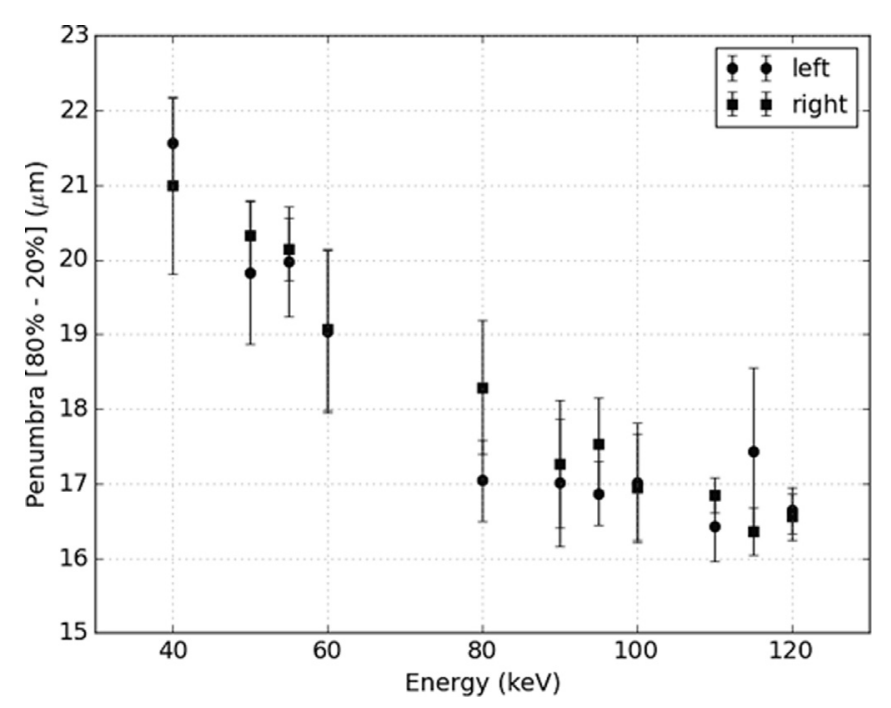

Fig. 7. The width of the microbeam penumbra as a function of monochromatic X-ray energy. The penumbra has been defined as the distance between $20 \%$ and $80 \%$ of the peak dose of the central microbeam. The labels "left" and "right" refer to the side of the microbeams that the penumbra was measured, according to beam's eye view.

obtained at each energy. The uncertainty represents a standard deviation of the mean. The penumbra are given for each side of the microbeams, although the same values, within uncertainty, are obtained for both sides. Penumbra measured using pink beam microbeam profiles were consistent with those measured using monochromatic beam profiles and have been excluded from the graph. The penumbra is observed to decrease with increasing energy until $\sim 90 \mathrm{keV}$, above which it appears to remain constant. Above $90 \mathrm{keV}$, the mean penumbra is $17 \pm 5 \mu \mathrm{m}$.

The effect of the presence of bone on the PVDR is demonstrated in Fig. 8. As in [31], a power equation has been fitted to the data obtained in the absence of bone material. Close to the bone-water interface, the PVDR is higher than in a homogeneous water phantom at the same depth. However, at depths greater than $\sim 18 \mathrm{~mm}$, the bone has little to no effect on the PVDR.

\section{Discussion}

A PTW microDiamond 60019 detector has been used to measure

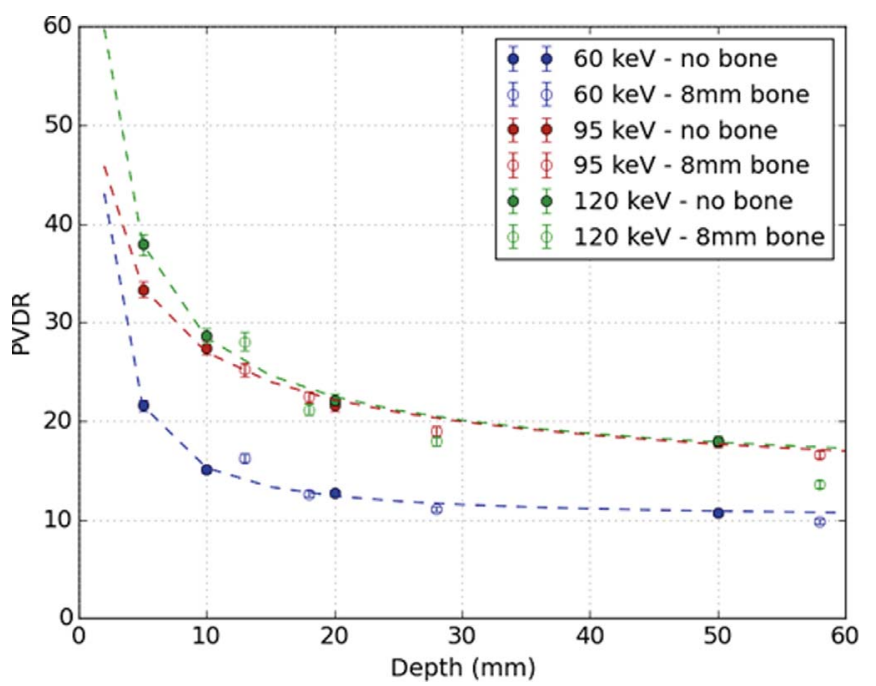

Fig. 8. PVDR as a function of depth in a water tank for monochromatic beams of 60,95 and $120 \mathrm{keV}$. For data acquired in the presence of bone, the thickness of the bone equivalent material $(8 \mathrm{~mm})$ has been taken into account in the depth. 
Table 2

PVDR as a function of depth for a field size of $20 \times 20 \mathrm{~mm}^{2}$ compared to PVDRs measured in similar conditions reported in the literature. Uncertainties correspond to 2 standard deviations of the mean to be comparable with Martínez-Rovira et al.[34].

\begin{tabular}{|c|c|c|c|c|c|c|}
\hline \multirow[t]{3}{*}{ Depth (mm) } & \multicolumn{6}{|l|}{ PVDR } \\
\hline & \multicolumn{2}{|c|}{ Martínez-Rovira et al.[34] } & \multirow[t]{2}{*}{ This work $\left(100 \mathrm{keV}^{\mathrm{b}}\right)$} & \multicolumn{2}{|c|}{ Livingstone et al. $[31]^{\mathrm{c}}$} & \multirow[t]{2}{*}{ This work $(95 \mathrm{keV})$} \\
\hline & Monte Carlo & Film & & microDiamond & Film & \\
\hline 5 & $42 \pm 3$ & $38 \pm 6$ & $37 \pm 1$ & $34 \pm 3$ & $34 \pm 7$ & $33 \pm 2$ \\
\hline 10 & $33 \pm 3$ & $33 \pm 5$ & $31 \pm 1$ & $28 \pm 2$ & $29 \pm 6$ & $28 \pm 4$ \\
\hline 20 & $28 \pm 2$ & $28 \pm 4$ & $25 \pm 1$ & $26 \pm 2$ & $22 \pm 5$ & $24 \pm 4$ \\
\hline 40 & $25 \pm 2$ & $22 \pm 3$ & - & - & $20 \pm 5$ & $21 \pm 2$ \\
\hline 50 & - & - & $21 \pm 1$ & $21 \pm 2$ & $21 \pm 6$ & $21 \pm 1$ \\
\hline 100 & $23 \pm 3$ & $19 \pm 3$ & - & $19 \pm 2$ & - & $19 \pm 1$ \\
\hline
\end{tabular}

\footnotetext{
a $100 \mathrm{keV}$ average energy spectrum.

${ }^{\mathrm{b}}$ Monochromatic.

c $95 \mathrm{keV}$ average energy spectrum.

d $95 \mathrm{keV}$ average energy spectrum.
}

experimentally the energy dependence of the peak to valley dose ratio with the goal of determining the optimal energy range for future clinical trials in MRT. Monochromatic $\mathrm{x}$-rays in the range $40-120 \mathrm{keV}$ were used to demonstrate the energy dependence of the PVDR. It was found that the PVDR increases with increasing energy prior to reaching a plateau at $\sim 100 \mathrm{keV}$. Unfortunately it is not possible to reach energies higher than $120 \mathrm{keV}$ using the monochromator installed on IMBL, however, a simulation study by Prezado et al. [27] investigating the PVDR as a function of beam energy (51-200 keV) demonstrated that the PVDR increases with increasing energy with a maximum PVDR at $175 \mathrm{keV}$. Another study by Shinohara et al. [28] suggested that the PVDR remains relatively constant with energy up to $300 \mathrm{keV}$ but decreases significantly with energy above this. Siegbahn et al.[20] showed that the valley dose comes mostly from secondary electrons which travel away from the peak where they were created before depositing their energy. As the energy and range of secondary electrons both increase with increasing primary photon energy, it is expected that the PVDR will decrease with increasing energy due to the relatively higher valley dose. This does not explain the initial increase of PVDR with increasing energy. Siegbahn et al.[20] also simulated the angular distribution of electrons leaving the planar microbeam and demonstrated that the peak angle of secondary electrons is decreasing with energy from 50 to $100 \mathrm{keV}$. This means that at $100 \mathrm{keV}$, secondary electrons are scattered in a more forward direction compared to at $50 \mathrm{keV}$ which may explain the initial increase in PVDR with increasing energy. In this study it was also demonstrated that the penumbra of the microbeams is decreasing with increasing energy before reaching a plateau at $\sim 90 \mathrm{keV}$. This supports the hypothesis that at low energies (below $90 \mathrm{keV}$ ), the contribution of lateral electrons is relatively significant compared to energies $100-120 \mathrm{keV}$.

Livingstone et al.[31] demonstrated that the microDiamond detector exhibits an energy response in the energy range studied in this work $(40-120 \mathrm{keV})$. The beam quality correction factor $\left(k_{Q}\right)$ found for the same microDiamond detector used in this study varies between $1.02 \pm 0.06$ at $120 \mathrm{keV}$ and $1.15 \pm 0.09$ at $40 \mathrm{keV}$. For a $95 \mathrm{keV}$ mean energy pink beam, the mean energy of the valley spectrum for the IMBL microbeam collimator is $15-20 \mathrm{keV}$ lower than that of the peak dose spectrum (depending on field size and depth of measurement), corresponding to an increase of $2 \%-3 \%$ in the beam quality correction required for the valleys compared to that for the peaks [31]. This would result in a decrease in the PVDR by the same amount. No similar published data exists for other energies, but if the same difference is assumed, the change in PVDR is within the experimental uncertainty given. Also, given that $k_{Q}$ increases with decreasing beam energy, even if the absolute values of the PVDR change, the observed trend and conclusion do not. Livingstone et al.[31] also showed that the microDiamond detector response is independent of dose rate in the range
$1-700 \mathrm{~Gy} / \mathrm{s}$, which encompasses the dose rates encountered in this study. Therefore no dose rate correction was applied.

Although it was demonstrated that $95 \mathrm{keV}$ monochromatic x-rays and a spectrum with $95 \mathrm{keV}$ weighted average energy have a similar PDD, monochromatic synchrotron X-ray beams have a flux which is several orders of magnitude lower than a spectrum with equivalent average energy. For this reason, monochromatic X-ray beams are not as suitable for MRT as pink beams. However, as the monochromatic beam is easily tuned between 20 and $120 \mathrm{keV}$ and there are restricted options to change the spectrum of the beam, monochromatic beams are extremely useful in this case for demonstrating energy dependence. Compared to monochromatic x-rays, the spectral x-rays with equivalent or similar average energy have similar PVDRs near the surface of a homogeneous water phantom, but have larger values at the larger depths due to hardening of the spectrum. This also makes spectral $\mathrm{x}$ rays more suitable for the treatment of deep-seated tumours using MRT. The PVDR as a function of depth for $95 \mathrm{keV}$ average energy are in agreement with those previously measured under the same conditions at IMBL [31] and are similar to those measured for the same microbeam geometry and similar spectrum (mean energy $99 \mathrm{keV}$ ) at the biomedical beamline (ID17) of the European Synchrotron Radiation Facility [34], as shown in Table 2 .

For simulating a more clinically relevant case, a slab of boneequivalent material with a thickness of $8 \mathrm{~mm}$ was placed in front of the water tank. This thickness is consistent with the frontal region of an adult male skull. When comparing the PVDR vs. depth curve measured in a homogeneous water tank to that measured in the head phantom at different energies, it was shown that although the PVDR is increased near the bone-water interface, with depth the difference decreases. At depths greater than $20 \mathrm{~mm}$, there is very little to no difference in the PVDRs measured with and without bone for 60 and $95 \mathrm{keV}$ x-rays. This trend was also observed by Martínez-Rovira et al. [35], although the actual PVDR values differ because the simulation considered the $99 \mathrm{keV}$ average energy of the ID17 spectrum instead of monochromatic x-rays and the thickness of bone was slightly greater $(10 \mathrm{~mm}$ compared to $8 \mathrm{~mm}$ ). The same trend was not observed for $120 \mathrm{keV}$ x-rays, but an investigation of the effect of bone at greater energies is required before discussing this point further. Since at IMBL the maximum monochromatic energy is $120 \mathrm{keV}$, a complementary Monte Carlo study would be required.

When taking into account PVDRs and microbeam penumbra measured in monochromatic beams, it is clear that the optimal energy range for MRT at IMBL is $100-120 \mathrm{keV}$. In this energy range or even lower, the bone makes little to no difference for the PVDR. This is important as radioresistant brain tumours have been identified as a clinical target for MRT. The maximum PVDRs were measured using the $124 \mathrm{keV}$ average energy spectrum. The optimal range found in this study fits within the 
recommended range of $100-300 \mathrm{keV}$ by Shinohara et al.[28]. Prezado et al.[27] found that the optimal energy for MRT was $175 \mathrm{keV}$, which is higher than what can be achieved at IMBL. Although these other studies indicate that higher energies are suitable for MRT not only due to the PVDR but the greater ability to penetrate tissue and deliver a therapeutic dose to a deep-seated tumour, it should be noted that for synchrotron wiggler generated X-ray beams, the photon flux decreases quite quickly with increasing energy above the peak energy as seen in Fig. 1. Indeed the beam can be filtered to shift the peak to higher energies, i.e., harden the beam, however this also decreases the integrated flux. As an example, for the spectrum with weighted average energy of $124 \mathrm{keV}$, for which the highest PVDR was measured, the integrated flux is $\sim 9$ times lower than for the spectrum with weighted average energy of $95 \mathrm{keV}$ [30]. For MRT a high dose rate is required in order to minimise tissue motion artifacts, therefore, the optimal energy for MRT will likely be a compromise between PVDR, beam penetration and dose rate to be determined on a case-by-case basis. There are possibilities to increase the flux, which include moving the sample stage closer to the wiggler source and increasing the wiggler magnetic field [30]. The latter is perhaps preferable since it also causes beam hardening, and thus improving or at worst maintaining the PVDR as well as increasing beam penetration. Increasing the wiggler field also decreases horizontal roll-off effects, meaning that the beam is more uniform for a given width. Furthermore, the beam decreases in size as the distance from the wiggler is decreased so the distance at which a patient can be treated is limited due to the size of the tumour.

\section{Conclusions}

The aim of this study was to experimentally optimise the X-ray energy for future clinical trials in MRT. Parameters such as percentage depth dose, peak to valley dose ratio and microbeam penumbra width as a function of energy were studied. A human head, including $8 \mathrm{~mm}$ of bone equivalent material, was simulated to determine the effect of bone on the PVDR at depth. In terms of PVDR and microbeam penumbra, it is recommended that the X-ray energy exceed $90 \mathrm{keV}$. The maximum PVDR was found at $124 \mathrm{keV}$ average energy, however, for IMBL the flux at this energy is likely to be too low for radiotherapy applications. A good compromise between PVDR, microbeam penumbra and flux was demonstrated at $95 \mathrm{keV}$. The presence of bone material was found to have little effect on the PVDR at depths beyond $20 \mathrm{~mm}$ for the energies studied.

\section{Conflict of Interest}

The authors have no conflict of interest to disclose.

\section{Acknowledgements}

This research was undertaken on the Imaging and Medical Beamline at the Australian Synchrotron, part of ANSTO. The authors thank the accelerator operators for beam availability. The authors also acknowledge financial support from LabEx PRIMES (ANR-11-LABX-0063/ANR11-IDEX-0007), COST TD1205 and NHMRC.

\section{References}

[1] Schültke E, Balosso J, Breslin T, Cavaletti G, Djonov V, Esteve F, et al. Microbeam radiation therapy: grid therapy and beyond. A clinical perspective. Br J Radiol 2017. http://dx.doi.org/10.1259/bjr.20170073. Epub ahead of print.

[2] Mohiuddin M, Fujita M, Regine WF, Megooni AS, Ibbott GS, Ahmed MM. High-dose spatially-fractionated Radiation (GRID): a new paradigm in the management of advanced cancers. Int J Radiat Oncol Biol Phys 1999;45(3):721-7.

[3] Ha JK, Zhang G, Naqvi SA, Regine WF, Yu CX. Feasibility of delivering grid therapy using a multileaf collimator. Med Phys 2005;33(1):76-82.

[4] Shirato H, Gupta NK, Jordan TJ, Henry JH. Lack of late skin necrosis in man after high-dose irradiation using small field sizes. Br J Radiol 1990;63(755):871-4.

[5] Zeman W, Curtis HJ, Baker CP. Histopathologic effecit of high-energy-particle mmicrobeam on the visual cortex of the mouse brain. Radiat Res 1961;15:496-514.
[6] Curtis HJ. The use of a deuteron microbeam for simulating the biological effects of heavy cosmic-ray particles. Radiat Res Suppl 1967;7:250-7.

[7] Slatkin DN, Spanne P, Dilmanian FA, Gebbers J-O, Laissue JA. Subacute neuropathological effects of microplanar bbeam of x-rays from a synchrotron wiggler. Proc Natl Acad Sci USA 1995;92:8783-7.

[8] Laissue JA, Geiser G, Spanne PO, Dilmanian FA, Gebbers JO, Geiser M, et al. Neuropathology of ablation of rat gliosarcoma and contiguous brain tissues using a microplanar beam of synchrotron-wiggler-generated x-rays. Int J Cancer 1998;78:654-60.

[9] Laissue JA, Lyubimova N, Wagner HP, Archer DW, Slatkin DN, Di Michiel M, et al. Microbeam radiation therapy. Proc. SPIE. 3770, medical applications of penetrating radiation. 1999. p. 38-45.

[10] Serduc R, van de Looij Y, Francony G, Verdonck O, van der Sanden B, Laissue J, et al. Characterization and quantification of cerebral edema induced by synchrotron x-ray microbeam radiation therapy. Phys Med Biol 2008;53:1153-66.

[11] Serduc R, Bräuer-Krisch E, Siegbahn EA, Bouchet A, Pouyatos B, Carron R, et al. High-precision radiosurgical dose delivery by interlaced microbeam arrays of highflux low-energy synchrotron X-rays. PLoS ONE 2010;5(2):e9028.

[12] Bräuer-Krisch E, Serduc R, Siegbahn EA, Le Duc G, Prezado Y, Bravin A, et al. Effects of pulsed, spatially fractionated microscopic synchrotron X-ray bbeam on normal and tumoral brain tissue. Mutat Res 2010;704:160-6.

[13] Bouchet A, Lemasson B, Duc GL, Maisin C, Bräuer-Krisch E, Siegbahn EA, et al. Preferential effect of synchrotron microbeam radiation therapy on intracerebral 9L gliosarcoma vascular networks. Int J Radiat Oncol Biol Phys 2011;78(5):1503-12.

[14] Bouchet A, Lemasson B, Christen T, Potez M, Rome C, Coquery N, et al. Synchrotron microbeam Radiation therapy induces hypoxia in intracerebral gliosarcoma but not in the normal brain. Radiother Oncol 2013;108(1):143-8.

[15] Bouchet A, Serduc R, Laissue JA, Djonov V. Effects of microbeam radiation therapy on normal and tumoral blood vessels. Phys Med 2015;31(6):634-41.

[16] Laissue JA, Blattmann H, Di Michiel M, Slatkin DN, Lyubimova N, Guzman R, et al. Weaning piglet cerebellum: a surrogate for tolerance to MRT (microbeam radiation therapy) in pediatric neuro-oncology. Proc. SPIE. 4508, penetrating radiation systems and applications II I. 2001. p. 65-73.

[17] Laissue JA, Blattmann H, Wagner HP, Grotzer MA, Slatkin DN. Prospects for microbeam radiation therapy of brain tumours in children to reduce neurological sequelae. Dev Med Child Neurol 2007;49:577-81.

[18] Grotzer MA, Schültke E, Bräuer-Krisch E, Laissue JA. Microbeam radiation therapy: clinical perspectives. Phys Med 2015;31(6):564-7.

[19] Bräuer-Krisch E, Bravin A, Zhang L, Siegbahn E, Stepanek J, Blattmann H, Slatkin DN, Gebbers JO, Jasmin M, Laissue JA. Characterization of a tungsten/gas multislit collimator for microbeam radiation therapy at the European Synchrotron Radiation Facility. Rev Sci Instrum 2005;76:064303.

[20] Siegbahn EA, Stepanek J, Bräuer-Krisch E, Bravin A. Determination of dosimetrical quantities used in microbeam radiation therapy (MRT) with Monte Carlo simulations. Med Phys 2006;33:3248-59.

[21] Serduc R, Bouchet A, Bräuer-Krisch E, Laissue JA, Spiga J, Sarun S, et al. Synchrotron microbeam radiation therapy for rat brain tumor palliation - influence of the microbeam width at constant valley dose. Phys Med Biol 2009;54:6711-24.

[22] Martínez-Rovira I, Sempau J, Fernández-Varea JM, Bravin A, Prezado Y. Monte Carlo dosimetry for forthcoming clinical trials in $\mathrm{x}$-ray microbeam radiation therapy. Phys Med Biol 2010;55:4375-88.

[23] Ibahim MJ, Crosbie JC, Yang Y, Zaitseva M, Stevenson AW, Rogers PAW, Paiva P. An evaluation of dose equivalence between synchrotron Microbeam Radiation Therapy and conventional broadbeam radiation using clonogenic and cell impedance assays. PLoS ONE 2014;9(6):e100547.

[24] Stepanek J, Blattmann H, Laissue JA, Lyubimova N, Di Michiel M, Slatkin DN. Physics study of microbeam radiation therapy with PSI-version of Monte Carlo code GEANT as a new computational tool. Med Phys 2000;27(7):1664-75.

[25] De Felici M, Felici R, Sanchez del Rio M, Ferrero C, Bacarian T, Dilmanian FA. Dose distribution from X-ray microbeam arrays applied to radiation therapy: an EGS4 Monte Carlo study. Med Phys 2005;32:2455-63.

[26] Spiga J, Siegbahn EA, Bräuer-Krisch E, Randaccio P, Bravin A. The GEANT4 toolkit for microdosimetry calculations: application to microbeam radiation therapy (MRT). Med Phys 2007;34:4322-30.

[27] Prezado Y, Fois G, Le Duc G, Bravin A. Gadolinium dose enhancement studies in microbeam radiation therapy. Med Phys 2009;36(8):3568-74.

[28] Shinohara K, Kondoh T, Nariyama N, Fujita H, Washio M, Aoki Y. Optimization of $\mathrm{X}$-ray microplanaar beam radiation therapy for deep-seated ttumor by a simulation study. J X-ray Sci Technol 2014;22:395-406.

[29] Livingstone J, Adam J-F, Crosbie J, Hall C, Lye J, McKinlay J, et al. Preclinical radiotherapy at the Australian Synchrotron's Imaging and Medical Beamline: Instrumentation, dosimetry and a small animal feasibility study. J Synchrotron Rad 2017;24(4):854-65.

[30] Stevenson AW, Crosbie JC, Hall CJ, Häusermann D, Livingstone J, Lye JE. Quantitative characterisation of the X-ray beam at the Australian Synchrotron Imaging and Medical Beamline (IMBL). J Synchrotron Rad 2017;24:110-41.

[31] Livingstone J, Stevenson AW, Butler DJ, Häusermann D, Adam JF. Characterization of a synthetic single crystal diamond detector for dosimetry in spatially fractionated synchrotron X-ray fields. Med Phys 2016;43(7):4283-93.

[32] Nath R, Biggs PJ, Bova FJ, Ling CC, Purdy JA, van de Geijn J, Weinhous MS. AAPM Code of practice for radiotherapy accelerators: report of AAPM radiation therapy task group 45. Med Phys 1994;21(7):1093-121.

[33] Lynnerup N. Cranial thickness in relation to age, sex and general body build in a Danish forensic sample. Forensic Sci Int 2001;117:45-51.

[34] Martínez-Rovira I, Sempau J, Prezado Y. Development and commissioning of a Monte Carlo photon beam model for the forthcoming clinical trials in microbeam radiation therapy. Med Phys 2012;39(1):119-31.

[35] Martínez-Rovira I, Sempau J, Prezado Y. Monte Carlo-based treatment planning system calculation engine for microbeam radiation therapy. Med Phys 2012;39(5):2829-38. 